\title{
On the analytic continuation of holomorphic solutions of partial differential equations
}

\author{
Jan Persson
}

\section{Introduction}

This note is a complement of an earlier paper by the author [8] on the local analytic continuation of holomorphic solutions of partial differential equations with holomorphic coefficients in $\mathbf{C}^{n}$. It is written in the light of some new results on the uniqueness in the local Cauchy problem, Persson [10]. See also Persson [11] for the connected question of $P$-convexity. The use of uniqueness cones in [10] made it possible to prove that some conditions are not only necessary but also sufficient for local uniqueness in the Cauchy problem. Since the cones of analytic continuation of [8] are counterparts of the uniqueness cones of [7] one may try the same way solving questions of analytic continuation.

The operators of [10] are defined in $\mathbf{R}^{3}$ and have constant coefficients. One proves that a certain geometric condition on the initial surface is necessary and sufficient. The point around which the Cauchy problem is posed is supposed to be simply characteristic with respect to the given differential operator $P$. The initial surface is supposed to be in $C^{2}$. The simplest nontrivial case of local analytic continuation is the problem in $\mathbf{C}^{2}$ when the coefficients of the operator are constants. Theorem 1.1 below treats this case. It turns out that one can give a simple necessary and sufficient geometric condition securing that a holomorphic solution can always be continued over the boundary at a given point. The boundary is supposed to be in $C^{1}$ and there is no restriction on the multiplicity if the point is characteristic.

Theorem 1.2 below is a restatement of $[8$, Theorem 4.1] concerning the existence of singular solutions. Theorem 1.3 is a reformulation of Theorem 1.2 giving a sufficient condition for the existence of solutions which cannot be continued analytically to the other side of the boundary. Theorem 1.1 shows that in $\mathbf{C}^{2}$ this condition is also necessary when the coefficients are constant. Theorem 1.4 extends Theorem 1.1 to the variable coefficients case as long as the boundary point is of the same kind as those in the constant coefficients case. 
The theorems are now stated and partly proved before their connection with earlier results are discussed. As to the notation one lets $z=\left(z_{1}, \ldots, z_{n}\right)=x+i y \in \mathbf{C}^{n}$, $x, y \in \mathbf{R}^{n}$. Let $D_{j}=\partial / \partial z_{j}=\left(\partial / \partial x_{j}-i \partial / \partial y_{j}\right) / 2$. The usual multi-index notation will be used. Let $\Omega \subset \mathbf{C}^{n}$ be an open set and let $H(\Omega)$ be the set of holomorphic functions in $\Omega$.

Definition 1.1. Let $\Omega \subset C^{n}$ be open and let $P(z, D)$ be a linear partial differential operator of order $m>0$ with holomorphic coefficients in $\Omega$. It is assumed that $P(z, D) \neq 0$ for all $z$ in $\Omega$. Let $F \subset \mathbf{C}^{n}$ be a closed set. Let $z^{0} \in \partial F \cap \Omega$. Assume that for each open set $\Omega^{\prime} \subset \Omega$ with $z^{0} \in \Omega^{\prime}$ the following is true. There exists an open set $\Omega^{\prime \prime} \subset \Omega^{\prime}, z^{0} \in \Omega^{\prime \prime}$ such that if $f \in H\left(\Omega^{\prime}\right), u \in H\left(\Omega^{\prime} \backslash F\right)$ fulfills $P(z, D) u=f$ in $\Omega^{\prime} \backslash F$ then there is a $v \in H\left(\Omega^{\prime \prime}\right)$ such that $u=v$ in $\Omega^{\prime \prime} \backslash F$ and $P(z, D) v=f$ in $\Omega^{\prime \prime}$. Then $z^{0}$ is called a point of local analytic continuation for $P$.

Remark. By the use of the Cauchy-Kovalevskij theorem around $z^{0}$ one can always reduce the problem of analytic continuation to that with $f=0$ if the order of $P$ at $z^{0}$ is $m$.

Theorem 1.1. Let $\Omega \subset \mathbf{C}^{2}$ be an open set and let $F \subset \mathbf{C}^{2}$ be closed. Let $P(D)$ be a linear partial differential operator with constant coefficients. Let $z^{0} \in \partial F \cap \Omega$. Let $\Omega^{\prime}$ be an open neighbourhood of $z^{0}$. Let $\varphi$ be a real valued function in $C^{1}\left(\Omega^{\prime}\right)$ such that after a rotation of coordinates $\operatorname{grad} \varphi\left(z^{0}\right)=(1,0)$ and such that $\Omega^{\prime} \backslash F=$ $\{z ; \varphi(z)<0\}$. Let $P_{m}$ be the principal part of $P$. Then $z^{0}$ is not a point of local analytic continuation if an only if $P_{m}((1,0))=0$ and there is an open set $\Omega^{\prime \prime} \subset \Omega^{\prime}, z^{0} \in \Omega^{\prime \prime}$ such that

$$
\varphi\left(z_{1}^{0}, z_{2}\right) \geqq 0, \quad\left(z_{1}^{0}, z_{2}\right) \in \Omega^{\prime \prime} .
$$

The proof will follow from Theorem 1.3 on one hand and from the use of cones of analytic continuation on the other hand. This last part of the proof will be given in Section 3.

The following theorem is proved in [8],

Theorem 1.2. (Persson [8, Theorem 4.1].) Let $m>0$ and $l, 0 \leqq l<m$ be integers and let $\beta=(l, m-l, 0, \ldots, 0) \in \mathbf{R}^{n}$. The linear partial differential operator $P(z, D)$ of order $m$ is given by

$$
P(z, D)=D^{\beta}-\sum_{\substack{|\alpha|=m \\ \alpha \neq \beta \\ \alpha_{1} \leqq l}} a_{\alpha} D^{\alpha}-\sum_{|\alpha|<m} a_{\alpha} D^{\alpha} .
$$

The coefficients $a_{\alpha}$ are holomorphic around the origin in $\mathbf{C}^{n}$. Let $g\left(z_{1}\right)$ be holomorphic in $\left\{z_{1} ; 0<\left|z_{1}\right|<c\right\}$ for some $c>0$. Then there exist a $c^{\prime}>0$ and a function $u$ holomorphic in $\left\{z ; 0<|z|<c^{\prime}, z_{1} \notin[0,+\infty)\right\}$ and satisfying

$$
P(z, D) u=0, \quad \text { and } \quad u(z)=g\left(z_{1}\right), \quad z_{2}=0 .
$$


One could also use Persson [9, Theorem 1] in the proof of Theorem 1.1 since the coefficients of $P$ are constants. For an earlier weaker version in the constant coefficient case see Kiselman [4, Lemme 5.6]. A version of Theorem 1.2 in the simply characteristic case has been proved by Kawai [3] and Tsuno [12, Theorem 2]. As to the connection between this type of theorems and hyperbolicity see [9] and Komatsu [5, Theorem 2 and Theorem 3].

In [8] the explicit reformulation of Theorem 1.2 as a theorem on analytic continuation is missing. It could be given as follows.

Theorem 1.3. Let $\Omega, P(z, D), z^{0}$ and $F$ be as in Definition 1.1. Let $\Omega^{\prime}$ be a neighbourhood of $z^{0}$ and let $\varphi \in \mathbf{C}^{\mathbf{1}}\left(\Omega^{\prime}\right)$ be real valued. It is assumed that

$$
\left\{z ; \varphi(z)<0, z \in \Omega^{\prime}\right\}=\Omega^{\backslash} \backslash F .
$$

It is assumed that after $a$ translation and rotation of coordinates $z^{0}=0$, and $\operatorname{grad}_{z} \varphi\left(z^{0}\right)=(1,0, \ldots, 0)$ and also that after multiplication by $\left(a_{\beta}\right)^{-1} P$ has the form (1.2). Then $z^{0}$ is not a point of local analytic continuation if for some neighbourhood $\Omega^{\prime \prime}$ of $z^{0}$

$$
\varphi\left(0, z^{\prime}\right) \geqq 0, \quad\left(0, z^{\prime}\right) \in \Omega^{\prime \prime}, \quad z^{\prime}=\left(z_{2}, \ldots, z_{n}\right) \in \mathbf{C}^{n-1} .
$$

Proof. It is clear from $(1.4),(1.5)$ and $\operatorname{grad}_{z} \varphi(0)=(1,0, \ldots, 0)$ that

$$
\varphi\left(x_{1}, z^{\prime}\right) \geqq 0, \quad\left(x_{1}, z^{\prime}\right) \in \Omega^{\prime \prime}, \quad x_{1} \geqq 0 .
$$

for $\Omega^{\prime \prime}=\{z ;|z|<c\}$ with $c^{\prime}>0$ small, and that $\left(x_{1}, z^{\prime}\right) \in F$ when $\varphi\left(x_{1}, z^{\prime}\right) \geqq 0$. Let $g\left(z_{1}\right)=z_{1}^{-1}$ in Theorem 1.2. Then $u$ of $(1.3)$ is in $H\left(\Omega^{\prime \prime} \backslash F\right)$ and cannot be continued analytically to a neighbourhood of $z^{0}$. The theorem is proved.

First part of the proof of Theorem 1.1. Let $P_{m}((1,0))=0$ and let $(1.1)$ be true. Then in the chosen coordinates $P$ must have the form (1.2) for some integer $l$, $0 \leqq l<m$, after a multiplication by a constant $c \neq 0$. Theorem 1.3 now tells that $z^{0}$ is not a point of local analytic continuation. The last part of the proof will be given in Section 3.

The proof of Theorem 1.1 will also give the following theorem.

Theorem 1.4. Let the hypothesis be as in Theorem 1.3 with the restriction that $n=2$. Then (1.5) is also a necessary condition for $z^{0}$ not to be a point of local analytic continuation.

For the conclusion of the proof see Section 3.

The cones of analytic continuation used in Section 3 differs somewhat in their definition from those used in [8]. It is therefore convenient to give a selfcontained treatment here. This is done in Section 2. This section starts with a proof of the linear Cauchy_Kovalevskij theorem in a precise form. The proof is an adaption 
of the proof of the same theorem in a special case due to Yamanaka in Yamanaka, Persson [19, Theorem 2.1]. Theorem 2.1 is a sharper version of Bony-Schapira [2], Corollaire] also due to $\mathrm{C}$. Wagschal who used majorant series for its proof.

Theorem 2.2 is a reformulation of Theorem 2.1. It is a sharper formulation of the fundamental observation made by Zerner [20] on the analytic continuation across a noncharacteristic border in $C^{1}$. This observation is used in [1], [2], and [8] and is founded on a remark by J. Schauder on the proof of the Cauchy-Kovalevskij theorem. See [2].

After the redefinition of cones of analytic continuation in Section 2 Theorem 2.3 and Theorem 2.4 follow giving sufficient conditions for a point to be a point of local analytic continuation. Theorem 2.4 is a correction of [8, Theorem 2.2]. See Section 2. Theorem 2.4 is a generalization of Bony-Schapira [2, Theorem 4.1]. One notices that Theorem 2.4 can be applied in some cases when the cone condition of BonySchapira is not fulfilled.

The papers by Tsuno [12]-- [18] contains a long range of results on analytic continuation. If these results can be specialized down to the situation treated in [8] or here then they are strictly weaker. But it should also be noticed that [12] is a forerunner of [8]. As to the results on analytic continuation in Pallu de la Barrière [6] it seems likely that at least the examples given on analytic continuation across the sphere and across a real hyperplane also could be proved by use of cones of analytic continuation. The example where there is no analytic continuation across a simply characteristic point on the sphere follows from Theorem 1.3. Otherwise the highly technical formulation of the theorems in [6] makes it difficult to compare them with the results in Tsuno [12]-[18], Persson [8] or in this note. It should be stressed that there are no results giving a sufficient and necessary condition for a characteristic point to be a point of local analytic continuation in [6] or [12]-[18]. However in Section 4 there is a theorem Theorem 4.1 giving a sufficient condition for a simply characteristic point to be a point of local analytic continuation. In the $\mathbf{C}^{2}$ case this condition is also necessary as Theorem 1.4 shows. Theorem 4.1 is a slight sharpening of Persson [8, Theorem 3.1]. For an earlier weaker version see Tsuno [12, Theorem 1]. The possibility of extending the existence results of [2] or [6] has not been investigated here.

It is likely that one will have difficulties also for very slight extensions of the results of the present note as long as one wants a necessary and sufficient condition. This seems to be the case for second order operators in $\mathbf{C}^{\mathbf{3}}$ at simply characteristic points when the border is in $C^{2}$. For comparison see [10] and [11]. 


\section{The Cauchy-Kovalevskij theorem and cones of analytic continuation}

Here one lets $\left|z^{\prime}\right|_{\infty}=\max _{2 \leqq j \leqq n}\left|z_{j}\right|, z^{\prime} \in \mathbf{C}^{n-1}$.

Theorem 2.1. (Yamanaka-Persson [19, Theorem 2.1].) Let $M>0$, and $T>0$ be constants and let $c \in \mathbf{C}^{n}$. Let $\beta=(m, 0, \ldots, 0) \in \mathbf{R}^{n}$ with $m>0$ an integer. Let $z^{\prime}=\left(z_{2}, \ldots, z_{n}\right) \in \mathbf{C}^{n-1}$ and $z=\left(z_{1}, z^{\prime}\right) \in \mathbf{C}^{n}$. Let $a_{\alpha},|\alpha| \leqq m$ be holomorphic and bounded in the set

Let

$$
S=\left\{z ;\left|z^{\prime}-c^{\prime}\right|_{\infty}<M,\left|z_{1}-c_{1}\right|<T\right\} .
$$

$$
A_{\alpha}=\sup _{S}\left|a_{\alpha}(z)\right| \text {, and } L=m e \max _{1 \leqq j \leqq m}\left(\sum_{\substack{\left|\alpha^{\prime}\right|=j \\ \alpha \mid=m}} \alpha^{\prime} ! A_{\alpha}\right)^{1 / j} \text {. }
$$

Let $f$ be holomorphic and bounded in $S$. Then there exists a unique holomorphic solution $u$ of

$$
D_{\mathbf{1}}^{m} u+\sum_{\substack{\alpha \mid \leq m \\ \alpha \neq \beta}} D_{1}^{\alpha_{1}} D^{\prime \alpha^{\prime}} a_{\alpha} u=f, \quad u=O\left(z_{1}^{m}\right)
$$

in $S_{L}=\left\{z ;\left|z^{\prime}-c^{\prime}\right|_{\infty}<M-L\left|z_{1}-c_{1}\right|, z \in S\right\}$.

Remark. In [19, Theorem 2.1] one has some non-zero initial data and $f=0$. Here the more general framing of Theorem 2.1 is prefered. The case with holomorphic non-zero initial data in $\left|z^{\prime}-c^{\prime}\right|_{\infty}<M$ can always be reduced to the Theorem 2.1 case with a new $f$ in $H(S)$. The new $f$ may not be bounded but the proof will show that this condition on $f$ in the hypothesis of Theorem 2.1 is superfluous since $f$ is bounded on compact subsets of $S$. In fact when the proof is completed one sees that it suffices to assume that $a_{\alpha} \in H\left(S_{L}\right),|\alpha| \leqq m, f \in H\left(S_{L}\right)$ with $a_{\alpha}$ bounded as before. One also notices that (2.2) is equivalent to $D_{1}^{m} u+\sum_{\substack{|\alpha| \leqq m \\ \alpha \neq \beta}} b_{\alpha} D^{\alpha} u=f$, $u=O\left(z_{1}^{m}\right)$ for some holomorphic functions $b_{\alpha}$ with $b_{\alpha}=a_{\alpha},|\alpha|=m$, and vice versa. One notices that $S_{L}$ only depends on the coefficients of the principal part of $P$.

Proof. It is no restriction to assume that $c=0$. This is done from now on Let $g$ be a holomorphic function around $z=0$. Let

$$
D_{1}^{-1} g(z)=\int_{0}^{z_{1}} g\left(s, z^{\prime}\right) d s
$$

where the integration is taken radially from 0 to $z_{1}$ in $\mathbf{C}$. Let $v=D_{1}^{m} u$. Then (2.2) is equivalent to

Let $v_{0}=f$ and let

$$
v=-\sum_{\alpha \neq \beta} D_{1}^{-m+\alpha} D^{\prime \alpha^{\prime}} a_{\alpha} v+f \text {. }
$$

The goal is to prove that $v=\sum_{j=0}^{\infty} v_{j}$ is a holomorphic solution of (2.3) in $S_{L}$. The following lemma will be used. 
Lemma 2.1. Let $A$ and $B$ be open subsets of $\mathbf{C}^{n-1}$ such that $A \subset B$ and such that the distance between $A$ and $\left[B\right.$ in the ||$_{\infty}$ norm is not less than a number $r>0$. Let $\mathrm{g}$ be bounded in $H(B)$ and let

$$
|g|_{D}=\sup _{j \in D}|g(z)|, \quad D \subset B .
$$

Then for any multi-index $\alpha$ the inequality

$$
\left|D^{\alpha} g\right|_{A} \leqq \alpha ! r^{-|\alpha|}|g|_{B}
$$

holds.

The proof is immediate from the Cauchy formula. One lets

$$
|w|_{r}=\sup \left\{\left|w\left(z^{\prime}\right)\right| ;\left|z^{\prime}\right|_{\infty}<M-r\right\}, \quad 0 \leqq r \leqq M,
$$

for $w$ holomorphic and bounded in $\left|z^{\prime}\right|<M$. At first assume that $L>0$. Let

It is noticed that

$$
K=1+\max _{j}\left(m e L^{-1}\right)^{j} \sum_{\substack{|\alpha|<m \\ \alpha_{1}=m-j}} \alpha^{\prime} ! A_{\alpha} .
$$

$$
K^{j} \geqq K, \quad \text { and } \quad h^{-\left|\alpha^{\prime}\right| \leqq h^{-j+1}+1, h>0, \quad\left|\alpha^{\prime}\right| \leqq j-1, \quad j=2,3, \ldots .}
$$

Let $w\left(z^{\prime}\right)$ be holomorphic and bounded in $\left|z^{\prime}\right|<M$. Then Lemma 2.1, (2.1) and (2.3) give

$$
\begin{gathered}
\sum_{\substack{|\alpha| \leqq m \\
\alpha_{1}=m-j}}\left|D^{\prime \alpha^{\prime}}\left(a_{\alpha} w\right)\right|_{r} \leqq|w|_{r-h}\left(\sum_{\substack{|\alpha|=m \\
\alpha_{1}=m-j}}+\sum_{\substack{|\alpha|<m \\
\alpha_{1}=m-j}}\right) \alpha^{\prime} ! h^{-\left|\alpha^{\prime}\right|} A_{\alpha} \\
{\left[\leqq|w|_{r-h}(L / m e)^{j}\left(h^{-j}+\left(h^{-j+1}+1\right) K\right)\right]_{j \geqq 2} \leqq|w|_{r-h}(L / m e)^{j}\left(h^{-1}+K\right)^{j},} \\
0 \leqq r-h<r \leqq M,
\end{gathered}
$$

Let $N=|f|_{s}$. It follows from (2.4) and (2.8) that

$$
\begin{gathered}
\left|v_{1}\left(z_{1}, \cdot\right)\right|_{r}=\sum_{\substack{|\alpha| \leqq m \\
\alpha_{1}=m-j}} D_{1}^{-m+\alpha_{1}} D^{\prime \alpha^{\prime}} a_{\alpha}\left(z_{1}, \cdot\right) f\left(z_{1}, \cdot\right)||_{r} \\
\leqq N \sum_{j=1}^{m}(L / m e)^{j}\left(r^{-1}+K\right)^{j}\left|z_{1}\right|^{j} / j !, \quad 0<r \leqq M, \quad\left|z_{1}\right|<T .
\end{gathered}
$$

It is now asserted that

$\left(\left|v_{p}\right|_{r} \leqq N m^{p-1} \sum_{j=p}^{m p}(L / m e)^{j}(p / r+K)^{j}\left|z_{1}\right|^{j} / j !, \quad 0<r \leqq M, \quad\left|z_{1}\right|<T, \quad p=1,2, \ldots\right.$ Now (2.9) says that (2.10) is true for $p=1$. Let (2.10) be true for $p=k$. Then (2.4) and $(2.10)$ give

$$
\begin{gathered}
\left|v_{k+1}\right|_{r} \leqq\left.\sum_{j=1}^{m}(L / m e)^{j}((k+1) / r+K)^{j}\left|D_{1}^{-j}\right| v_{k}\left(z_{1}, \cdot\right)\right|_{k r /(k+1)} \mid \\
\leqq N m^{k-1} \sum_{j=1}^{m}(L / m e)^{j}((k+1) / r+K)^{j} \sum_{l=k}^{k m}(L / m e)^{l}((k+1) / r+K)^{l}\left|z_{1}\right|^{l+j} /(l+j) ! \\
\left.=N m^{k-1} \sum_{j=1}^{m} \sum_{l=k}^{k m}(L / m e)^{l+j}(k+1) / r+K\right)^{l+j}\left|z_{1}\right|^{j+l} /(j+l) ! \\
\leqq N m^{k} \sum_{j=k+1}^{(k+1) m}(L / m e)^{j}((k+1) / r+K)^{j}\left|z_{1}\right|^{j} / j !, \quad\left|z_{1}\right|<T, \quad 0<r \leqq M .
\end{gathered}
$$

So $(2.10)$ is true for all $p$. 
One shall now use

$$
(1+K r / p)^{m p}<e^{m K r}, \quad p=1,2, \ldots,
$$

and the fact that there exists a constant $x$ independent of $p$ such that

$$
p^{j} / j ! \leqq x e^{j}, \quad j=p, p+1, \ldots, \quad p=1,2, \ldots .
$$

One notices that

$$
\sum_{j=p}^{m p}\left(L\left|z_{1}\right| / r\right)^{j} \leqq m p\left(L\left|z_{1}\right| / r\right)^{p}, \quad\left|z_{1}\right| \leqq r / L, \quad p=1,2, \ldots
$$

It follows from (2.10)-(2.13) that

$$
\begin{gathered}
\left|v_{p}\left(z_{1}, \cdot\right)\right|_{r} \leqq N m^{p-1} \sum_{j=p}^{m p} m^{-j}\left(L\left|z_{1}\right| / r\right)^{j}(1+K r / p)^{j} e^{-j} p^{j} / j ! \\
\leqq N \varkappa e^{m K r} p\left(L\left|z_{1}\right| / r\right)^{p}, \quad\left|z_{1}\right|<r / L, \quad p=1,2, \ldots
\end{gathered}
$$

It follows from (2.14) that $v=\sum_{p=1}^{\infty} v_{p}$ converges uniformly on compact subsets of $S_{L}$ and thus is holomorphic. Now (2.4) and $v_{0}=f$ shows that $v$ solves (2.3) and that $u=D_{1}^{-m} v$ solves (2.2) in $S_{L}$.

If $L=0$ then one replaces this $L$ with another $L>0$ in the computations above. Since this $L$ is arbitrary the solution $u$ exists in all $S=S_{0}$. Of course this depends on the uniqueness of the solution. But this follows from the uniqueness of the formal power series solution of (2.2). Theorem 2.1 is proved.

Remark. In the following it is refered to (2.1) as if it gives $L>0$. This is achieved if one lets $L=0$ be replaced by $L=1$ keeping $L$ unchanged in the other cases. This is done for technical reasons.

Theorem. 2.2 Let $z=x+i y, x, y \in \mathbf{R}^{n}$. Let $\Omega \subset \mathbf{C}^{n}$ be an open convex set. Let $m>0$ be an integer and let $\beta=(m, 0, \ldots, 0) \in \mathbf{R}^{n}$. Let $a_{\alpha} \in H(\Omega),|\alpha| \leqq m$ be bounded and let $a_{\beta}=1$. Define $P(z, D)=\sum_{|\alpha| \leqq m} a_{\alpha} D^{\alpha}$. For $r>0$ define

and

$$
S(r)=\left\{z ; x_{1}=0,\left|y_{1}\right|<r,\left|z^{\prime}\right|_{\infty}<r\right\}
$$

$$
K(r)=\left\{z ;\left|y_{1}\right|<r,\left|z^{\prime}\right|_{\infty}<r-L\left|x_{1}\right|\right\} .
$$

Here the constant $L>0$ is defined by (2.1) with $S=\Omega$. Let $V \subset \Omega$ be an open convex set and let $u \in H(V)$ and $f \in H(K(r))$. If $S(r) \subset V, K(r) \subset \Omega$, and if $P(z, D) u=f$ in $V$ then there is a $v \in H(K(r))$ satisfying $P(z, D) v=f$ in $K(r)$ and $u=v$ in $V \cap K(r)$.

Proof. Let $\left|y_{1}^{0}\right|<r$. Then the initial data $D_{1}^{j} u\left(y_{1}^{0}, z^{\prime}\right), 0 \leqq j<m$, exist and are holomorphic in $\left|z^{\prime}\right|_{\infty}<r$. It follows from Theorem 2.1 that there exists a solution $v$ of $P(z, D) v=f$ with these initial data in

$$
\left\{z ;\left|z^{\prime}-z^{0}\right|_{\infty}<r-L\left|z_{1}-i y_{1}^{0}\right| .\right.
$$

Especially $v$ exists and is holomorphic in $K(r)$. The theorem is proved. 
Definition 2.1. Let $\Omega \subset \mathbf{C}^{n}$ be an open set. Let $M$ be an open convex cone in $\mathbf{C}^{n}$ with vertex at the origin. Let $N \in M$ be such that

$$
M \subset\{z ; \operatorname{Re}\langle z, N>0\rangle\} \text {. }
$$

Here $\langle z, N\rangle=z_{1} N_{1}+\ldots+z_{n} N_{n}$. It is assumed that $P(z, D)$ is a linear partial differential operator with coefficients holomorphic in $\Omega$. Its order is $m>0$ and $P_{m}(z, D)$ is its ptincipal part. Let $z^{0} \in \Omega$ and let $G \subset C^{n}$ be a closed convex set such that $z^{0}$ is an inner point of $G$. One defines

$$
K\left(N, M, z^{0}, G\right)=\left\{z: z \in G, \operatorname{Re}\left\langle z-z^{0}, \delta\right\rangle\langle z, N>0\rangle \leqq 0, \delta \in M\right\} .
$$

If $K\left(N, M, z^{0}, G\right)$ is compact, if there is an open set $U \subset \Omega$ such that $K\left(N, M, z^{0}, G\right) \subset U$, and if $P_{m}(z, \delta) \neq \neq 0, z \in U, \delta \in M$, then $K\left(N, M, z^{0}, G\right)$ is called a cone of analytic continuation at $z^{0}$ for the operator $P$.

Remark. If one identifies $z=x+i y \in \mathbf{C}^{n}$ with $\left(x_{1}, y_{1}, x_{2}, y_{2}, \ldots, x_{n}, y_{n}\right) \in \mathbf{R}^{2 n}$ one should identify $N$ with the vector $\left(\operatorname{Re} N_{1},-\operatorname{Im} N_{1}, \ldots, \operatorname{Re} N_{n},-\operatorname{Im} N_{n}\right.$ ) when one uses the usual scalar product in $\mathbf{R}^{2 n}$.

The following theorem is a slight generalization of [8, Theorem 2.1].

Theorem 2.3. Let $\Omega, U, P(z, D)$ and $K\left(N, M, z^{0}, G\right)$ be as in Definition 2.1. Let $V \subset \Omega$ be an open set such that

$$
K\left(N, M, z^{0}, G\right) \cap \partial G \subset V .
$$

Let $f \in H(U)$ and $u \in H(V)$ such that $P(z, D) u=f$ in $U \cap V$. Then there are an open set $W \subset U$ such that $K\left(N, M, z^{0}, G\right) \subset W$ and $a v \in H(W)$ such that $P(z, D) v=f$ in $W$ and $v=u$ in a neighbourhood of $K\left(N, M, z^{0}, G\right) \cap \partial G$. This neighbourhood and $W$ are independent of $u$ and $f$.

Proof. Let $d(A, B)$ denote the distance between to sets $A$ and $B$ in $\mathbf{C}^{n}$. Choose $\varepsilon>0$ so small that $d\left(K\left(N, M, z^{0}, G\right) \cap \partial G,\lceil V)>2 \varepsilon\right.$. Define

$$
K(\varepsilon)=\left\{z ; z \in \partial G, d\left(\{z\}, K\left(N, M, z^{0}, G\right) \cap \partial G\right)\right\} \leqq \varepsilon .
$$

Let $\varepsilon^{\prime}>0$ and define

$$
S^{\prime}\left(\varepsilon^{\prime}\right)=\left\{z ; z-z^{0} \mid \leqq \varepsilon^{\prime}\right\}
$$

Then one defines $K\left(\varepsilon, \varepsilon^{\prime}\right)$ as the convex hull of $K(\varepsilon)$ and $S^{\prime}\left(\varepsilon^{\prime}\right)$. One notices that $z^{0}$ is an inner point of the convex set $G$. Thus by first choosing $\varepsilon$ and then $\varepsilon^{\prime}$ it is possible to achieve that $K\left(\varepsilon, \varepsilon^{\prime}\right) \subset U, K(\varepsilon) \subset V$ and that at each point $z \in \partial K\left(\varepsilon, \varepsilon^{\prime}\right) \cap$ $\left\lceil\partial G\right.$ there is a closed ball $B \subset K\left(\varepsilon, \varepsilon^{\prime}\right) \cup\left(V \cap U \cap\left\{K\left(\varepsilon, \varepsilon^{\prime}\right)\right)\right.$ of radius $\varepsilon^{\prime}$ with $z \in \partial B$ and the normal $N^{0}$ of $B$ at $z$ being noncharacteristic. As to $N^{0}$ see the remark after Definition 2.1. Let $\varepsilon$ and $\varepsilon^{\prime}$ be chosen as above. Choose an open ball $B_{1} \subset U \cap G$ with centre at $z$. This is always possible since $z$ has a positive distance to $\partial G$. Let $f \in H(U)$ and let $u \in H\left(B_{1} \cap\left(\dot{K} \varepsilon, \varepsilon^{\prime}\right)\right)$ fulfilling $P(z, D) u=f$ in $B_{1} \cap \stackrel{K}{K}\left(\varepsilon, \varepsilon^{\prime}\right)$. After 
a rotation and translation of the coordinate system one achieves that $z=0$ and $N^{0}=(1,0, \ldots, 0) \in \mathbf{C}^{n}$. And perhaps after choosing the radius of $B_{1}$ still smaller and after multiplication of the differential equation by a $g \in H\left(B_{1}\right), g(z) \neq 0 \quad z \in B_{1}$, one assumes that $P(z, D)=\sum a_{\alpha} D^{\alpha}$ with $a_{\alpha}$ all bounded and in $H\left(B_{1}\right)$ and with $a_{(m, 0, \ldots, 0)}=1$ in $B_{1}$. Choose $r, 0<r<\varepsilon^{\prime}$. Let $K(r)$ and $S(r)$ be as in Theorem 2.2. Let $L$ be defined by (2.1) with $S=B_{1}$. It is now obvious that one may choose $r$ so small that $S(r)+(-r / 2 L, 0, \ldots, 0) \subset B_{1} \cap \stackrel{\circ}{B}$ and $K(r)+(-r / 2 L, 0, \ldots, 0) \subset B_{1}$. A translation of the result of Theorem 2.2 gives that $u$ can be continued analytically to $K(r)+(-r / 2 L, 0, \ldots, 0)$ which is a neighbourhood of $z=0$.

The argument above will now be used to conclude the proof. Let $f$ and $u$ be as in the hypothesis of the theorem. Choose a fixed $z \in K\left(N, M, z^{0}, G\right) \cap \partial G$. Define

$$
K_{t}=K\left(\varepsilon, \varepsilon^{\prime}\right)+\left\{(1-t)\left(z-z^{0}\right)\right\}, \quad 0 \leqq t \leqq 1
$$

If $\varepsilon^{\prime}$ is small then $u$ and $f$ are holomorphic in a neighbourhood of $K_{0} \cap G$. Let $t^{\prime}$ be the least upper bound of thoose $t$ for which there is a $v$ holomorphic and equal to $u$ in a neighbourhood of $K_{t} \cap \partial G$ and holomorphic in the inner points of $K_{t} \cap G$ fulfilling $P(z, D) v=f$ there. If $t^{\prime}=1$ then the proof is concluded. One just chooses $W$ as the union of the inner points of $K_{1}=K\left(\varepsilon, \varepsilon^{\prime}\right)$ and a neighbourhood of $K_{1} \cap \partial G$ where $u=v$. If $t^{\prime}<1$ then the argument in the preceeding paragraph shows that $t^{\prime}$ is an inner point of the set for which it is the least upper bound. So $t^{\prime}<1$ is impossible. The proof is concluded.

Theorem 2.4. Let $\Omega$ and $P(z, D)$ be as in Theorem 2.3. Let $F \subset C^{n}$ be a closed set and let $z^{0} \in \partial F \cap \Omega$. Then $z^{0}$ is a point of local analytic continuation if to each open set $\Omega^{\prime} \subset \Omega$, with $z^{0} \in \Omega^{\prime}$ there is a cone of analytic continuation $K\left(N, M, z^{0}, G\right) \subset \Omega^{\prime}$

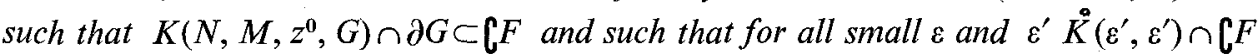
is connected. Here $K\left(\varepsilon, \varepsilon^{\prime}\right)$ is defined in the proof of Theorem 2.3.

Proof. Let $f \in H\left(\Omega^{\prime}\right)$ and $u \in H\left(\Omega^{\prime} \backslash F\right)$ such that $P(z, D) u=f$ in $\Omega^{\prime} \backslash F$. Theorem 2.3 or rather its proof shows that for sufficiently small $\varepsilon$ and $\varepsilon^{\prime}$ there exist a open neighbourhood $W$ of $K\left(\varepsilon, \varepsilon^{\prime}\right)$ and a $v \in H(W)$ fulfilling $P(z, D) v=f$ in $W$ and $v=u$ in a neighbourhood $V \subset W$ of $K\left(\varepsilon, \varepsilon^{\prime}\right) \cap \partial G$. Since $\dot{K}\left(\varepsilon, \varepsilon^{\prime}\right) \cap \complement F$ is connected one must have $u=v$ there. Choose $\stackrel{\circ}{K}\left(\varepsilon, \varepsilon^{\prime}\right) \cup V$ as $\Omega^{\prime \prime}$ in Definition 1.1. Theorem 2.4 is proved.

Remark. One notices that the topological error in [8, Theorem 2.2] does not intervene in the proof of [8, Theorem 3.1] since the connectedness condition of Theorem 2.4 is always satisfied in the application of [8, Theorem 2.2] in [8, Section 3]. 


\section{Proof of Theorem 1.1 and Theorem 1.4. The last part}

After a rotation and translation of the coordinates in Theorem 1.1 one may assume that $z^{0}=0$ and that $\operatorname{grad}_{z} \varphi(0)=(1,0)$. If $P_{m}((1,0)) \neq 0$ then one can always find and open convex cone $M$ of noncharacteristic directions in $\mathbf{C}^{2}$ containing $N=(1,0)$ and such that $K(N, M, 0, G)$ is a cone of analytic continuation if $G$ is a closed convex set with $O \in G \dot{G}$. Let $G=\left\{z ; x_{1} \geqq-\varepsilon\right\}$. Then it is obvious that $K(N, M, 0, G) \cap \partial G \subset B \backslash F$ if $\Omega^{\prime}$ is a fixed neighbourhood of $z=0$ and $B \subset \Omega^{\prime}$ is an open small ball around 0 and if $\varepsilon>0$ is chosen small after $B$ being chosen. Theorem 2.4 says that $z=0$ is a point of analytic continuation. This fact is found already in Zerner [20]. Its proof is given here just to follow the line given in the introduction.

Let $P_{m}((1,0))=0$ and let $(1.1)$ of Theorem 1.1 be false. Then there is a sequence of complex numbers $\left(s_{j}\right)_{j=1}^{\infty}$ such that $s_{j} \rightarrow 0, j \rightarrow \infty$, and such that

$$
\varphi\left(\left(0, s_{j}\right)\right)<0, \quad j=1,2, \ldots .
$$

For convenience one assumes that for each $j$ one makes a rotation in the $z_{2}$ variable such that in the new variables all $s_{j}$ are real and positive. Still after a multiplication of $P$ by a constant $c$ with $|c|=1 P$ may be assumed to have the form in (1.2) where the absolute values of the coefficients are independent of $j$ in the new coordinate systems. This is important in the following.

Let

$$
M=\left\{t \zeta ; \zeta=\xi+i \eta, \xi, \eta \in \mathbf{R}^{2}, \xi_{1}=1,0<-\xi_{2}<b,\left|\eta_{j}\right|<b, j=1,2, t>0\right\}
$$

for some constant $b>0$. It follows from (1.2) that $P_{m}(\zeta) \neq 0, \zeta \in M$, when $b$ is small since then

$$
\left|\zeta_{1}^{m-l} \zeta_{2}^{l}\right|>\sum_{\substack{|\alpha|=m \\ \alpha_{2}<l}}\left|a_{\alpha} \zeta^{\alpha}\right|, \quad \zeta \in M
$$

One notices that this is true also in the Theorem 1.4 case when the coefficients are variable but bounded in a neighbourhood of $z^{0}$. Let $N=(1,-b / 2) \in \mathbf{C}^{2}$. Let $G$ be a closed convex set containing 0 as an inner point. Then for each small open ball $B$ around 0 and with any $G$ such that $K(N, M, 0, G) \subset B K(N, M, 0, G)$ is a cone of analytic continuation for $P$ at $z=0$.

For each $j$ define

$$
G_{j}=\left\{z ; x_{1} \geqq-\varepsilon_{j}, x_{2} \leqq s_{j}\right\}
$$

Here $\varepsilon_{j}>0$. One notices that

$$
K\left(N, M, 0, G_{j}\right) \subset\left\{z: x_{1} \geqq-\varepsilon_{j}, x_{2} \leqq s_{j}, x_{1} \leqq-b\left|y_{j}\right|, j=1,2, x_{1} \leqq b x_{2}\right\}
$$


It now follows from the fact that $\partial F$ is in $C^{1}$, from (3.1) and (3.3) that to each open ball $B$ around 0 one can choose $j$ and then $\varepsilon_{j}>0$ such that $K\left(N, M, 0, G_{j}\right) \cap$ $\partial G_{j} \subset B \backslash F, K\left(N, M, 0, G_{j}\right) \subset B$. It follows from Theorem 2.4 that 0 is a point of local analytic continuation. The same argument applies in the Theorem 1.4 case. The proofs of Theorem 1.1 and Theorem 1.4 are complete.

\section{Analytic continuation across a simply characteristic point}

In this section the following slight sharpening of Persson [8, Theorem 3.1] will be proved.

Theorem 4.1. Let $\Omega, P(z, D), z^{0}$ and $F$ be as in Definition 1.1. Assume tha ${ }^{t}$ for some open set $V \subset \Omega, z^{0} \in V$, there is a real valued $\varphi \in C^{2}(V)$ with $\operatorname{grad}_{z} \varphi\left(z^{0}\right) \neq 0$ such that

$$
V \cap F=\{z ; \varphi(z) \geqq 0, z \in V\}
$$

also fulfilling

$$
P_{m}\left(z^{0}, \operatorname{grad}_{z} \varphi\left(z^{0}\right)\right)=0, \text { and } \operatorname{grad}_{\zeta} P_{m}\left(z^{0}, \operatorname{grad}_{z} \varphi\left(z^{0}\right)\right) \neq 0
$$

Let $\mathbf{C} \ni t \rightarrow(z(t), \zeta(t)) \in \mathbf{C}^{2 n}$ solve

$$
\begin{gathered}
d(z(t), \zeta(t)) / d t=\left(\operatorname{grad}_{\zeta} P_{m}(z(t), \zeta(t)),-\operatorname{grad}_{z} P_{m}(z(t), \zeta(t))\right) \\
(z(0), \zeta(0))=\left(z^{0}, \operatorname{grad}_{z} \varphi\left(z^{0}\right)\right)
\end{gathered}
$$

If for some sequence $\left(t_{j}\right)_{j=1}^{\infty}, t_{j} \in \mathbf{C}, j=1,2, \ldots$ one has $t_{j} \rightarrow 0, j \rightarrow \infty$ and

$$
\varphi\left(z\left(t_{j}\right)\right)<0, \quad j=1,2, \ldots
$$

then $z^{0}$ is a point of local analytic continuation.

Proof. As is pointed out in the proof of [8, Theorem 3.1] (4.2) and (4.3) are invariant under holomorphic changes of coordinates. Of course this is true for (4.4) too. As in [8] one might assume that $z^{0}=0$

$$
\operatorname{grad}_{z} \varphi(0)=(1,0, \ldots, 0)
$$

and

$$
P_{m}(z, D)=D_{1}^{m-1} D_{2}+\sum_{\substack{\alpha_{1}<m-1 \\|\alpha|=m}} a_{\alpha} D^{\alpha}
$$


from the beginning. Then (4.3) gives $z(t)=(0, t, 0, \ldots, 0)$. So (4.4) is

$$
\varphi\left(\left(0, t_{j}, 0, \ldots, 0\right)\right)<0, \quad j=1,2, \ldots
$$

As in Section 3 for each $j$ one chooses new coordinates by rotating the $z_{2}$ variable and keeping the other variables fixed such that $t_{j}$ is real and positive. After multiplication of $P$ by a constant $c,|c|=1$ the new $P_{m}$ has still the form (4.6) and all absolute values of $a_{\alpha}$ are unchanged just as in Section 3. For a reader who accepts the proof of [8, Theorem 3.1] the proof of Theorem 4.1 is then complete. However the proof in [8] at this point is not selfcontained and refers to a proof in [7]. The idea is that one first shows that it is possible to continue $u$ analytically from the origin up to the line $\mathbf{R} \ni s \rightarrow z(s)$ to a certain positive distance from $z=0$ independent of $j$. For this one uses cones of analytic continuation just as uniqueness cones are used in the corresponding situation in [7]. Then (4.7) and the fact just proved makes it possible to insert a cone of analytic continuation with vertex at $z=0$ such that Theorem 2.4 can be applied showing that $z=0$ is a point of local analytic continuation.

As before one lets $z=x+i y, x, y \in \mathbf{R}^{n}$. One also lets $\zeta=\xi+i \eta, \xi, \eta \in \mathbf{R}^{n}, z^{\prime \prime}=$ $\left(z_{3}, \ldots, z_{n}\right) \in \mathbf{C}^{n-2}$ and $\zeta^{\prime \prime}=\left(\zeta_{3}, \ldots, \zeta_{n}\right) \in \mathbf{C}^{n-2}$. One also writes $z \zeta=z_{1} \zeta_{1}+\ldots+z_{n} \zeta$ and $z^{\prime \prime} \zeta^{\prime \prime}=z_{3} \zeta_{3}+\ldots+z_{n} \zeta_{n}$.

For each $j$ one constructs cones of analytic continuation as follows. Let $b>0$, and $c>0$ be constants. Let

$$
M_{1}=\left\{t \zeta ; \xi_{1}=1,\left(\eta_{1}^{2}+\eta_{2}^{2}+\left|\zeta^{\prime \prime}\right|^{2}\right)<-b^{2} \xi_{2}, 0<-\xi_{2}<c, t>0\right\}
$$

and

$$
M_{2}=\left\{t \zeta ; \xi_{1}=1,\left(\eta_{1}^{2}+\eta_{2}^{2}+\left|\zeta^{\prime \prime}\right|^{2}\right)<b^{2} \xi_{2}, 0<\xi_{2}<c, t>0\right\}
$$

and let $N_{1}=(1,-c / 2,0, \ldots, 0) \in \mathbf{C}^{n}$ and $N_{2}=(1, c / 2,0, \ldots, 0) \in \mathbf{C}^{n}$. It follows from (4.6) that for some small fixed $b$ and $c$ there is a neighbourhood $U$ of $z=0$ such that

$$
P_{m}(z, \zeta) \neq 0, \quad z \in U, \quad \zeta \in M_{k}, \quad k=1,2
$$

Let $G$ be a closed convex set such that $z^{0}$ is an inner point of $G$ and such that

$$
K\left(N_{k}, M_{k}, z^{0}, G\right) \subset U, \quad k=1,2 .
$$

That shows that for these $z^{0}$ and $G K\left(N_{k}, M_{k}, z^{0}, G\right)$ is a cone of analytic continuation for $P$ at $z^{0}$.

Let $z$ be fixed and such that $\sup \operatorname{Re} z \zeta \leqq 0, \zeta \in M_{1}$. That means that

$$
x_{1} \leqq-x_{2} \xi_{2}+y_{1} \eta_{1}+y_{2} \eta_{2}-\operatorname{Re} z^{\prime \prime} \zeta^{\prime \prime}, \quad \zeta \in M_{1}, \quad \xi_{1}=1 .
$$


Now (4.10) and (4.8) give

$$
x_{1} \leqq-x_{2} \xi_{2}-\left|\left(y_{1}, y_{2}, z^{\prime \prime}\right)\right| b\left|\xi_{2}\right|^{1 / 2}, \quad 0<-\xi_{2}<c,
$$

For $x_{2} \leqq 0 \quad(4.11)$ gives

$$
x_{1} \leqq c x_{2}-b c^{1 / 2}\left|\left(y_{1}, y_{2}, z^{\prime \prime}\right)\right|
$$

Let $x_{2}>0$ and define $g(t)=x_{2} t-\left|\left(y_{1}, y_{2}, z^{\prime \prime}\right)\right| b t^{1 / 2}, t \geqq 0$. Then inf $g(t)=$ $-4^{-1} b^{2} \mid\left(y_{1}, y_{2}, z^{\prime \prime} \mid 2 / x_{2}=g\left(4^{-1} b^{2}\left|\left(y_{1}, y_{2}, z^{\prime \prime}\right)\right|^{2} / x_{2}^{2}\right)\right.$. That means that for $\left|\left(y_{1}, y_{2}, z^{\prime \prime}\right)\right|<$ $2 b^{-1} c^{1 / 2} x_{2}$

$$
x_{1} \leqq-4^{-1} b^{2}\left|\left(y_{1}, y_{2}, z^{\prime \prime}\right)\right|^{2} / x_{2} .
$$

On the other hand for $x_{2}>0$ and $\left|\left(y_{1}, y_{2}, z^{\prime \prime}\right)\right| \geqq 2 b^{-1} c^{1 / 2} x_{2}(4.12)$ is true. It follows that

(4.14) $K\left(N_{1}, M_{1}, 0, G\right) \subset\left\{z ; \quad z \in G, x_{1} \leqq c x_{2}-b c^{1 / 2}\left|\left(y_{1}, y_{2}, z^{\prime \prime}\right)\right|, \quad\right.$ when $x_{2} \leqq 0$ or when $x_{2}>0$ and $\left|\left(y_{1}, y_{2}, z^{\prime \prime}\right)\right| \geqq 2 b^{-1} c^{1 / 2} x_{2}$, and $x_{1} \leqq-4^{-1} b^{2}\left|\left(y_{1}, y_{2}, z^{\prime \prime}\right)\right|^{2} / x_{2}$ when $x_{2}>0$ and $\left|\left(y_{1}, y_{2}, z^{\prime \prime}\right)\right|<2 b^{-1} c^{1 / 2} x_{2}$.

Let $z^{0}=\left(x_{1}^{0}, x_{2}^{0}, 0, \ldots, 0\right)$. By comparing $M_{1}$ and $M_{2}$ and the computations that gave (4.14) one sees that

$K\left(N_{2}, M_{2}, z^{0}, G\right) \subset\left\{z ; \quad z \in G, x_{1}-x_{1}^{0} \leqq-c\left(x_{2}-x_{2}^{0}\right)-b c^{1 / 2}\left|\left(y_{1}, y_{2}, z^{\prime \prime}\right)\right|\right.$ when $x_{2}-x_{2}^{0} \geqq 0$ or when $x_{2}-x_{2}^{0}<0$ and $\left|\left(y_{1}, y_{2}, z^{\prime \prime}\right)\right| \geqq-2 b^{-1} c^{1 / 2}\left(x_{2}-x_{2}^{0}\right)$, and $x_{1} \leqq 4^{-1} b^{2}\left|\left(y_{1}, y_{2}, z^{\prime \prime}\right)\right|^{2} /\left(x_{2}-x_{2}^{0}\right) \quad$ when $\quad x_{2}-x_{2}^{0}<0 \quad$ and $\quad\left|\left(y_{1}, y_{2}, z^{\prime \prime}\right)\right|<$ $-2 b^{-1} c^{1 / 2}\left(x_{2}-x_{2}^{0}\right)$.

One notices that the constants $b$ and $c$ are chosen independent of the special coordinate system chosen for a fixed $j$.

Since $\varphi \in C^{2}$ near $z=0$ and since $\operatorname{grad}_{z} \varphi(0)=(1,0, \ldots, 0)$ one may write $\varphi(z) \geqq 0$ as

$$
x_{1} \geqq g\left(y_{1}, z^{\prime}\right)=A\left(\left(y_{1}, x^{\prime} y^{\prime}\right)\right)+o\left(\left|\left(y_{1}, x^{\prime}, y^{\prime}\right)\right|^{2}\right) .
$$

Here $g \in C^{2}$ and $A$ is a homogeneous polynomial of order 2. Thus there exists a constant $K_{1}>0$ independent of $j$ such that

$$
\varphi(z)<0 \quad \text { if } \quad x_{1}<-K_{1}\left(y_{1}^{2}+\left|z^{\prime}\right|^{2}\right) .
$$

Choose $\varepsilon>0$ independent of $j$ and define $G=\left\{z ; x_{1} \geqq-\varepsilon, x_{2} \geqq 0\right\}$. Let $z^{0}=$ $(-s, t, 0, \ldots, 0), s>0, t>0$. It follows from (4.15)-(4.17) that

$$
\partial G \cap K\left(N_{2}, M_{2}, z^{0}, G\right) \subset\{z ; \varphi(z)<0\},
$$


for some small fixed $t$ and $\varepsilon$ and all small $s$ independent of $j$. Theorem 2.3 and the fact that $K\left(N_{2}, M_{2}, z^{0}, G\right) \cap\{z ; \varphi(z)<0\}$ is connected shows that $u$ can be continued to a neighbourhood of $K\left(N_{2}, M_{2}, z^{0}, G\right)$ for each small $s$. That means that for $z^{0}=(0, t, 0, \ldots, 0) u$ can be continued analytically to all inner points of $K\left(N_{2}, M_{2}, z^{0}, G\right)$. A look at (4.15) says that there is an $\varepsilon>0$ and a $K_{2}>0$ independent of $j$ such that $u$ can be continued analytically to

$$
\left\{z ; x_{1}<-K_{2}\left(y_{1}^{2}+y_{2}^{2}+\left|z^{\prime \prime}\right|^{2}\right), 0<x_{2}<t / 2, x_{1}>-\varepsilon\right\} \text {. }
$$

Let $\varepsilon_{j}>0$ and define

$$
G_{j}=\left\{z ; x_{1} \geqq-\varepsilon_{j}, x_{2} \leqq t_{j}\right\}
$$

It follows from (4.17), (4.14), (4.7) and (4.18) that $u$ can be continued analytically to a neighbourhood of

$$
\partial G_{j} \cap K\left(N_{1}, M_{1}, 0, G_{j}\right)
$$

if $j$ is big and $\varepsilon_{j}>0$ is chosen small. Theorem 2.4 shows that $z=0$ is a point of local analytic continuation. The proof of Theorem 4.2 is complete.

Added in proof. It should be noticed that the constructions in M. Kashiwara and P. Schapira, Problème de Cauchy pour les systèmes microdifférentiels dans le domaine complexe, Inventiones math. 46 (1978) 17-38, specialized to one differential eqation and solutions with singularities in a hypersurface give precisely Theorem 1.2. In Theorem 1.2 the $x_{2}$ direction is "non microcaractéristique,.. The proof in [8] shows that in this case the use of microdifferential operators is not essential.

\section{References}

1. BeNGel, G., Sur le prolongement analytique des solutions d'une équation aux dérivées partielles, C. R. Acad. Sci. Paris Sér. A -B 273 (1971), 572-574.

2. Bony, J.-M. et SChapira, P., Existence et prolongement des solutions holomorphes des équations aux dérivées partielles, Invent. Math. 17 (1972), 95-105.

3. KawaI, T., Construction of local elementary solutions for linear partial differential operators with real analytic coefficients I, Publ. RIMS Kyoto Univ. 7 (1971/72), 363-397.

4. Kiselman, C. O., Prolongement des solution d'une équation aux dérivées partielles à coefficients constants, Bull. Soc. Math. France, 97 (1969), 329-356.

5. Komatsu, H., Irregularity of characteristic elements and hyperbolicity, Publ. RIMS Kyoto Univ. 12, suppl., (1977), 233-245.

6. Pallu de la Barriére, Ph., Existence et prolongement des solutions holomorphes des équations aux dérivées partielles, J. Math. Pure Appl. 55 (1976), 21 -46.

7. Persson, J., On uniqueness cones, velocity cones and P-convexity, Ann. Mat. Pura Appl. (4) 96 (1973), 69-87. 
8. Persson, J., Local analytic continuation of holomorphic solutions of partial differential equations, Ann. Mat. Pura Appl. (4) 112 (1977), 193-204.

9. Persson, J., The Cauchy problem and Hadamard's example, J. Math. Anal. Appl. 59 (1977), $522-530$.

10. Persson, J., The Cauchy problem at simply characteristic points and $P$-convexity, Ann. Mat. Pura Appl. (4) 122 (1979) 117-140.

11. Persson, J., The wave operator and P-convexity, Boll. Un. Mat. Ital. (5) 18-B (1981) 591-604.

12. Tsuno, Y., On the prolongation of local holomorphic solutions of partial differential equations, J. Math. Soc. Japan 26 (1974), 523-548.

13. Tsuno, Y., On the prolongation of local holomorphic solutions of nonlinear partial differential equations, Proc. Japan Acad. 50 (1974), 702-705, J. Math. Soc. Japan 27 (1975), $454-466$.

14. Tsuno, Y., On the prolongation of local holomorphic solutions of partial differential equations, II, prolongation across the pluriharmonic hypersurface, J. Math. Soc. Japan 28 (1976), 304-306.

15. Tsuno, Y., On the prolongation of local holomorphic solutions of partial differential equations, III, equations of the Fuchsian type, J. Math. Soc. Japan 28 (1976), 611-616.

16. Tsuno, Y., Holomorphic continuation of solutions of degenerate partial differential equations in two variables, Proc. Japan Acad., Ser. A, 54 (1978), 226-229.

17. Tsuno, Y., Holomorphic continuation of solutions of partial differential equations across the multiple characteristic surface, J. Math. Soc. Japan 32 (1980), 286-299

18. Tsuno, Y., Localization of differential operators and holomorphic continuation of the solutions, Hirosima Math. J. 10 (1980) 539-551.

19. Yamanaka, T. and Persson, J., On an extension of Holmgren's uniqueness theorem, Coment. Math. Univ. St. Paul. 22 (1973), 19-30.

20. Zerner, M., Domaine d'holomorphie des fonctions vérifiant une équation aux dérivées partielles, C. R. Acad. Sci. Paris Sér. A-B 272 (1971), 1646-1648.

Received January 21, 1980

Jan Persson

Institutt for matematiske realfag

Universitetet i Tromsö

Breivika

Postboks 953

N-9001 TROMSÖ

Norway 